\title{
MedDRA High Level Group Term
}

National Cancer Institute

\section{Source}

National Cancer Institute. MedDRA High Level Group Term. NCI Thesaurus. Code C71889.

A superordinate descriptor for one or more MedDRA high level terms (HLT). It is subordinate to System Organ Class (SOC). 